\title{
On Finite Alexandroff Space Co-Topology Matrix
}

\author{
Clara K. Paluku \\ Département de Mathématique et Informatique \\ Université Pédagogique Nationale \\ Kinshasa, DR Congo
}

\begin{abstract}
This paper deals with Alexandroff spaces for which any topology always admits a co-topology. It is also shown that this characteristic makes Alexandroff spaces different from common topological spaces. Related theorems are demonstrated.
\end{abstract}

Keywords: Alexandroff space, topology, co-topology, family.

\section{INTRODUCTION}

In his article, Russian Mathematician Pavel Sergeevitch Alexandroff (1896 - 1982) has introduced the concept of « Diskrete Räume » or discret spaces today known as Alexandroff spaces [1].

In my article I put the relationship "topology - co-topology" wich makes Alexandroff spaces different from other topological spaces.

In fact, I show that a topology is a Alexandroff one if and if it has a co-topology (of which opens are the closed of the initial topology).

Let $\mathrm{X}=\{1,2, \ldots, p, \ldots, n\}$ a $n$ items set and let $\tau$ a topology onto $\mathrm{X}$. for any $\mathrm{p} \in \mathrm{X}, i$ definie $U_{p}$, the smallest (in the sense of the set inclusion) item of $\tau$ containing the p point. The $U_{p}$ form a base of $\tau$ called irreductible base.

The $\tau$ topology is stocked in the form of a binary squared matrix $M(\tau)=\left(a_{p q}\right)$ of $n$ order, where for any $p$ and $q$ in $\mathrm{X} a_{p q}=1$ if $q \in U_{p}$ and 0 if not.

At last, I show (demonstrate) that the matrix of co-topology is equal to the transposed of the matrix of topology.

\section{DEFINITIONS OF CONCEPTS}

Definion 1.1. (Alexandroff Space).A $X$ topological space is a Alexandroff space if and only if any open intersection is an open, i.e., for any $O_{i} \in \tau$, we have $\bigcap_{i \in I} O_{i} \in \tau$. Then any sum of closed of $X$ is a closed of $X$.

Discret topological spaces and finite topological spaces are all Alexandroff spaces.

Definion 1.2. (Co-topology).Let $\tau$ a topology onto the $\mathrm{X}$ set. Let $\tau^{*}$ the set of closed of $(\mathrm{X}, \tau)$, i.e., $\tau^{*}$ $=\{\mathrm{S} \subset \mathrm{X}: \mathrm{X} \backslash \mathrm{S} \in \tau\}$. If $\tau^{*}$ forms a topology onto $\mathrm{X}, \tau^{*}$ is called in this assumption the co-topology of $\tau$.

Example 1.1. Let $X=\{a, b, c, d\}$. consider the topology $\tau=\{\varnothing,\{a\}, X\}$ the set of the closed of that topology is $\tau^{*}=\{\varnothing,\{b, c, d\}, X\}$ which is also a topology onto $X$, therefore a co-topology of $\tau$.

In general, any topology onto a $\mathrm{X}$ finite set admits a co-topology. 


\section{Fundamental Theorems and Propositions}

Theorem 2.1. Let $\tau$ is a topology onto X. then $\tau$ is a Alexandroff topology if $\tau$ admits a co-topology onto X.

\section{Proof}

If $\tau$ is a Alexandroff topology onto $\mathrm{X}$ and $\tau^{*}=\{\mathrm{S} \mathrm{CX}: \mathrm{X} \backslash \mathrm{S} \in \zeta\}$ is the set of closed of $(\mathrm{X}, \tau)$, we have clearly that $\emptyset \in \tau^{*}$ by definition of $X \in \tau^{*}$ of $\varnothing \in \tau^{*}$. If $\mathrm{A}, \mathrm{B} \in \emptyset \in \tau^{*}$, then $\mathrm{X} \backslash \mathrm{A} \in \tau$ and $\mathrm{X} \backslash \mathrm{B} \in \tau$. Hence $(\mathrm{X} \backslash \mathrm{A}) \cup(\mathrm{X} \backslash \mathrm{B})=\mathrm{X}(\mathrm{A} \cap B) \in \tau$ (since $\tau$ is a topology, i.e., it means that) $\mathrm{A} \cap B \in \tau^{*}$. Equally if $A_{\propto} \in \tau^{*} \forall \alpha \in \Gamma$, then $\mathrm{X} \backslash A_{\alpha} \in \tau \forall_{a} \Gamma$.

As $\tau$ is a Allexandroff topology onto $\mathrm{X}$, it follows that $\cap\left\{\mathrm{X} \backslash A_{\alpha}: \propto \in \Gamma\right\}=\mathrm{X} \backslash\left(\mathrm{U}_{\alpha \in \Gamma} A_{\alpha}\right)$ belongs $\tau$. This shows (demonstrates) that $\mathrm{U}\left\{A_{\propto} / \propto \in \Gamma\right\} \in \tau^{*}$. Thus $\tau^{*}$ is a topology onto $\mathrm{X}$.

Conversely (reciprocally), assume that $\tau$ admit a $\tau^{*}$ co-topology. Show (demonstrate) that $\tau$ is a Alexandroff topology.

Let $\{\mathrm{Vi}: \mathrm{i} \in \mathrm{I}\}$ a set (family) of opens in $(\mathrm{X}, \tau)$, it must be show (demonstrated) that $\cap\left\{V_{i}: i \in I\right\}$ is an open in $(\mathrm{X}, \tau)$, that to say that its complementary is closed.

Trough DeMorgan laws we have: $\mathrm{X} \backslash\left(\cap\left\{V_{i}: i \in I\right\}\right)=\mathrm{U}(X \backslash) \in \tau^{*}$ since each $\mathrm{X} \backslash V_{i} \in \tau^{*}, \tau^{*}$ being a topology by hypothesis.

Hence $\mathrm{X} \backslash\left(\cap\left\{V_{i}: i \in I\right\}\right)$ is a closed in $(\mathrm{X}, \tau)$ and thus $\cap\left\{V_{i}: i \in I\right\}$ is an open in $(\mathrm{X}, \tau)$. This shows that $\tau$ is a Alexandroff topology.

Definition 2.1.Let $X$ a topological space and $p \in X$; we put Up = $\cap\{V \subset X: V$ inanopenof $X$ and $p \in V\}$. In other words $\mathrm{Up}$ is the smallest open containing $\mathrm{p}$.

Theorem 2.2.Let $X$ a topological space. Then $X$ is a Alexandroff space if and if $\{U p$ : and $p \in X\}$ is a base of X opens.

\section{Proof:}

Assume $\mathrm{X}$ a Alexandroff. Let $\mathrm{V}$ an open of $\mathrm{X}$ and $\mathrm{p} \in \mathrm{V}$.

By hypothesis $U p$ is an open of $X$ and it's the smallest open containing $p$. hence $p \in U p \subset V$. what shows (demonstrates) that $\{U p: p \in X\}$ is a base of opens of $X$.

Reciprocally, assume that the set (family) $\{U p: p \in X\}$ is a base of $X$ opens.

Let $\left\{V_{\alpha}: \in \Gamma\right\}$ a some family (set) of $\mathrm{X}$ opens. Put $\mathrm{V}=\cap\left\{V_{\alpha}: \alpha \in \Gamma\right\}$ and show (demonstrate) that $\mathrm{V}$ is an open of $\mathrm{X}$.

If $\mathrm{V}=\varnothing$, there is nothing to demonstrate. If no let $\mathrm{p} \in \mathrm{V}$.

Then $\mathrm{p} \in V_{\alpha}$ for any $\in \Gamma$. Hence $\mathrm{p} \in \mathrm{Up} \subset V_{\alpha} \forall \alpha \in \Gamma$ and thus $\mathrm{p} \in \mathrm{Up} \subset \mathrm{V}$. this shows (demonstrates) that $\mathrm{V}$ is next to each of its points, i.e., $\mathrm{V}$ is an open of $\mathrm{X}$.

Definition 2.2.Let $X$ a Alexandroff space. The family $\mathcal{B}=\{U p: p \in X\}$ is called the irreductible base of $\mathrm{X}$. this base is a characteristic of Alexandroff spaces.

Example 2.1. Let $X=\{a, b, c\}$ and $\tau=\{\varnothing,\{a\},\{a, c\},\{a, b, c\}\}$ a Alexandroff topology onto $X$.Then $\mathcal{B}=\{\mathrm{Ua}, \mathrm{Ub}, \mathrm{Uc}\}$ where $\mathrm{Ua}=\{\mathrm{a}\}, \mathrm{Ub}=\{\mathrm{a}, \mathrm{b}, \mathrm{c}\}$ et $\mathrm{Uc}=\{\mathrm{a}, \mathrm{c}\}$.

Proposition 2.1. Any Alexandroff space possesses an irreductible base.

\section{Proof}

If $X$ is a Alexandroff space, then family (the set) $\{U p: p \in X\}$ an irreductible base of $X$. 
Definition 2.3. Let $X=\{1,2, \ldots, p, \ldots$,$\} a n$ items finite set and let $\tau$ a Alexandroff topology onto X.X being finite, any $p$ item of $X$ has a proximity $U p=\cap\{V: V \subset X$ and $V$ is an open proximity of $p\}$. The family $\left\{U_{1}, U_{2}, \ldots, U_{p}, \ldots, U_{n}\right\}$ forms a base of open proximities of $(X, \tau)$.

The matrix of the space topology $(X, \zeta)$ is the binary squared matrix $\mathrm{M}(\zeta)=(\mathrm{apq})$ of $n$ order, where for any, $\mathrm{a}_{\mathrm{pq}}=1$ if $\mathrm{q} \in \mathrm{U}_{\mathrm{p}}$ and 0 if not.That matrix fully characterizes the $\tau$ topology.

In fact, as we know the matrix $\mathrm{M}(\tau)=\left(\mathrm{a}_{\mathrm{pq}}\right)$, we find all the items of each $\mathrm{U}_{\mathrm{p}}$ through the following rule $\mathrm{q} \in \mathrm{U}_{\mathrm{p}}$ if and if $\mathrm{a}_{\mathrm{pq}}=1$.

Example 2.2. Consider Alexandroff space $(\mathrm{X}, \tau)$, where $\mathrm{X}=\{\mathrm{a}, \mathrm{b}, \mathrm{c}\}$ and $\tau=\{\varnothing,\{\mathrm{a}\},\{\mathrm{a}, \mathrm{c}\}, \mathrm{X}\}$. The $\tau$ topologial matrix is given by

$$
M(\tau)=\left(\begin{array}{lll}
1 & 0 & 0 \\
1 & 1 & 1 \\
1 & 0 & 1
\end{array}\right) .
$$

Theorem 2.3.If $\left(\mathrm{a}_{\mathrm{pq}}\right)$ is the matrix of the topology of a finite Alexandroff space, then the matrix of the co-topology is the transposed $\left(a_{p q}\right)$.

\section{Proof}

Let $(\mathrm{X}, \tau)$ a finite Alexandroff space of which the digraph is written $\mathrm{D}(\tau)$, its adjacent matrix is defined by $\mathrm{A}(\mathrm{D}(\tau))=\beta_{i j}$ with

$$
\beta_{i j}=\left\{\begin{array}{c}
1 \operatorname{si}(i, j) \in D(\tau) e t i \neq j \\
0 \text { sinon }
\end{array}\right.
$$

The matrix $\mathrm{A}(\mathrm{D}(\zeta))$ meets the relation $\mathrm{A}(\mathrm{D}(\zeta))=\mathrm{M}(\zeta)$ - I where $\mathrm{I}$ is the any unit matrix and $\mathrm{M}(\zeta)$ the matrix of $\tau$ topology.

Let $\mathrm{D}\left(\tau^{*}\right)$ the diagraph of the co-topology, it is the opposite of the $\mathrm{D}(\zeta)$ diagraph of the $\zeta$ topology and $\mathrm{A}\left(\mathrm{D}\left(\tau^{*}\right)\right)$ is the transposed of $\mathrm{A}(\mathrm{D}(\tau))$.

So we have: $\mathrm{M}\left(\tau^{*}\right)=\mathrm{I}+\mathrm{A}^{\mathrm{T}}(\mathrm{D}(\tau))=\mathrm{M}^{\mathrm{T}}(\zeta)$ where $\mathrm{A}^{\mathrm{T}}(\mathrm{D}(\tau))$ and $\mathrm{M}^{\mathrm{T}}(\tau)$ respectively pointing the transposed of $\mathrm{A}(\mathrm{D}(\tau))$ matrix and the transposed of $\mathrm{M}(\tau)$. Thus $\mathrm{M}\left(\tau^{*}\right)=M^{T}(\tau)$.

Example 2.3.Considering the previous Alexandroff space $(X, \tau)$ where $X=\{a, b, c\}$, $\tau=\{\varnothing,\{a\},\{a, c\}, X\}$ and $\tau^{*}=\{\varnothing,\{b\},\{b, c\}, X\}$ the matrix of the co-topology is

$$
M\left(\tau^{*}\right)=\left(\begin{array}{lll}
1 & 1 & 1 \\
0 & 1 & 0 \\
0 & 1 & 1
\end{array}\right)
$$

\section{CONCLuding Remarks}

In this paper, I have made a clear relationship between topology and co-topology while dealing with Alexandroff spaces. I have also shown that this relationship makes Alexandroff spaces different from other topological spaces. Some theorems relating to the matter have been stated and demonstrated.

\section{ACKNOWLEDGEMENTS}

I am thankful to anonymous reviewer for constructive remarks on this paper and to Dr. Ruffin-Benoît M. Ngoie for helpful remarks and proofreading.

\section{REFERENCES}

[1]. AlexandroffP.S. 1937, DiskreteRäume, Mat.Sbornik, 501-518

[2]. Arenas F.G, 1999, Alexandroff space, Acta Math. Univ. Comemanae, 68(1): 17-25

[3]. Strong R.E. 1966, Finite topological spaces, Trans. AMS. (123): 325-340 


\section{Author's Biography}

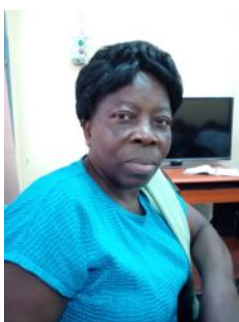

Clara K. Paluku is Senior Lecturer at Université Pédagogique Nationale (UPN) (Kinshasa, DR Congo). She is PhD. Student at the same University. Her main fields are General Topology, Algrebraic Topology, Functional Analysis and Algebra. 